\title{
The Effects of Modeling, Prompting, and Reinforcement in Deictic Gesture Instruction of a Student with Autism
}

\author{
JUNG-CHANG TANG \\ WAN-JU SHIH \\ National ChiaYi University, Taiwan
}

\begin{abstract}
The purpose of this study was to explore the effects of gesture instruction on the communication abilities of a student with autism. The multiple probe across settings of the single-case approach was conducted. The independent variable was gesture instruction, and the dependent variable was communication behaviour including the frequency of motoric gesture communication and deictic gesture communication. The participant accepted the experimental treatment of baseline, processing and maintenance phase one by one. Visual inspection was employed to assess the treatment effects of gesture instruction on this student. Results of this study showed that gesture instruction enhanced the frequency of the student's deictic gesture communication behaviour. The treatment effects could be maintained for two weeks and be generalized to another situation. Additionally, gesture instruction decreased the frequency of the student's motoric gesture communication behaviour and the treatment effects could be maintained for two weeks. After intervention, both teachers and parents interviewed indicated positive attitudes toward the gesture instruction, and confirmed the improvement on communication performance of this student.
\end{abstract}

\section{INTRODUCTION}

Gesture expressions, which develop very early in typical children, are one form of non-speech communication systems (Schopler \& Bristol, 1993). Even young infants could begin to use eye contact, vocalization, and gesture to communicate with others (Stone, Ousley, Yoder, Hogan, \& Hepburn, 1997). Such gestural or prelinguistic communication is a very important tool for infants and people with language impairment to make their needs understood 
before developing speech (Rosenquest, 2003). However, people with autism usually do not know how to use this form of communication to interact with others. Approximately $50 \%$ of children diagnosed with autism fail to develop such a mode of communication (Keen, Sigafoos, \& Woodyatt, 2005). One possible reason might be that they have difficulty in learning such deictic gestural forms through observation and imitation (Smith \& Bryson, 1998). This deficit in imitation skills may contribute to the difficulties individuals with autism have in using deictic gestural communication (Wetherby \& Prutting, 1984). Thus, even though they have difficulty speaking, rarely do they use functional gesture as an alternate means of communication (Wetherby \& Prutting, 1984). Because gestural behaviour plays an important role in the establishment and maintenance of social interactions, difficulties in this area might contribute to the difficulties individuals with autism have during social interactions (Garfin \& Lord, 1986; Koegel \& Frea, 1993). Therefore, teaching children with autism without speech alternative communication modes, such as deictic gesture expressions, in an effective manner become a crucial issue.

Children with autism are often nonverbal or lack communication expression skills (Chuang, 2001). Besides, they demonstrate difficulties in communicative initiations and social communicative behaviours (Hetzroni \& Shalem, 2005). Compared with children with Down's Syndrome and regular children, children with autism find it very difficult to make eye contact, to comprehend the meanings of gestures from others and to actively express themselves through simple gestures (Attwood, Frith, \& Hermelin, 1988). Studies have shown that the number of gestures used by individuals with autism is significantly lower than that used by others (Landry \& Loveland, 1989; Mundy, Sigman, \& Kasari, 1990; Sigman, Mundy, Sherman, \& Ungerer, 1986). More importantly, those gestures used by individuals with autism are typical of a lower level of development (Carr \& Kemp, 1989; Mundy, Sigman, Ungerer, \& Sherman, 1986). Children with autism often use instrumental or motoric gestures, like touching, contacting, and taking objects rather than distal gestures, such as pointing and showing deictic gestures to draw attention to their needs (Curcio, 1978; Loveland \& Landry, 1986). These observations are further confirmed by studies showing that children with autism have difficulties in producing or comprehending declarative but not imperative pointing (e. g., Baron-Cohen, 1989; Paparella \& Kasari, 
2004). Loveland and Landry (1986) even found that it is difficult for children with autism to comprehend the meaning of task stimuli when they are provided with gesture and verbal prompts. However, they also found that children with autism could perform better in linguistic tasks while given gesture prompts. Although research has defined the extent of the impairment in gestural communication, little research to date has demonstrated that gestural communication can be taught successfully to individuals with autism (Buffington, Krantz, McClannahan, \& Poulson, 1998; Carr \& Kemp, 1989). Due to a lack of research on teaching of gestural communication to children with autism, the current study focused on teaching deictic gestural responses. The purpose of this current study was to examine the efficacy of modeling, prompting, and reinforcement in deictic gestural instruction on communication behaviour of a student with autism.

\section{METHOD}

\section{Student and Setting}

The participant, Jamie was an eight-year-old boy and classified as autism spectrum disorders with absence of speech. Jamie studied in a self-contained class which included one teacher and one teacher assistant in a regular elementary school located in central Taiwan. He could recognize some concrete objects or items and took care of himself independently. However, he often showed poor attention and a lack of eye contact. His social emotion seemed unstable and he easily cries in typical situations. In addition, he never exhibited deictic gesture communication behaviour to express his needs. Instead, he would take objects directly without requesting permission.

\section{Measures}

The independent variable was the gesture instruction, and the dependent variable was the communication behaviour, including the frequency of motoric gesture communication and deictic gesture communication. Jamie's motoric gesture behaviour was defined as "nodding his head repetitively" or "taking preferred items directly without permission" while presenting preference items by the investigator. In contrast, his deictic gesture behaviour was defined as "pointing to the preferred items in a distance with his index finger 
and clasping the other fingers together" while presenting preference items. The investigator videotaped each condition using a videocassette recorder and a stopwatch. Two observers recorded the rates of communicative responses after presenting eight preferred items three times, respectively. All behavioural data were converted to percentage of frequency during which communicative behaviour occurred.

\section{Interobserver Agreement}

Before conducting the gestural communicative training, two graduate students of special education were trained for 5 hours to use the observational system and reached a $90 \%$ agreement criterion, and then served as observers for all sessions. These two observers recorded data independently and compared data sheets simultaneously. Across experiments, an average of $26 \%$ of the sessions (range, $20 \%$ to $34 \%$ ) was scored for interobserver agreement. An agreement was computed using an interval-byinterval agreement method to assess percentage agreement for the frequency of communicative behaviours (Kazdin, 1982). Interobserver agreement was computed by dividing the number of agreements by the number of agreements plus the number of disagreements and multiplying by 100\%. The interobserver agreement for Jamie's motoric gesture and deictic gesture behaviour is $92 \%(85 \%$ to $100 \%)$ and $94 \%(90 \%$ to $100 \%)$, respectively.

\section{Assessing Preference}

Before conducting gestural communicative training, object or edible preference ratings were determined by presenting five different kinds of objects or food/drink in a horizontal row. No instructions were given; the experimenter waited for the student to choose an item. The preference sessions began by seating the student with objects or food/drink in front of him. Five preassessment sessions were conducted. The student had free access to the preferred item for 30 minutes each session. Items for Jamie were chosen according to the reports of his classroom teachers. All items in Jamie's preference assessment consisted of manipulative toys or edibles. Preference was assessed using a multiple-stimulus without replacement (MSWO) procedure (DeLeon \& Iwata, 1996). The experimenter presented 5 items to Jamie in a linear array. Jamie was permitted to choose one item from the array. After a particular item was chosen, he had 10 s access to the item or to eat the food, after 
which time the trials resumed. This procedure continued until all items were chosen, or until no choice was made. This procedure was repeated 3 times. Preference was determined as the percentage of times an item was selected. The more preferred items were incorporated into gestural communication training during the treatment evaluation phase. A single subject methodology was then used to detect the treatment effects for such communication training. The research design and procedure for conducting gestural instructions are addressed below.

\section{Research Design}

A multiple probe across settings design was used to assess the effects of gestural communication training on Jamie's motoric gesture and deictic gesture behaviour. The frequency with communicative behaviour was the dependent variable. Gestural communicative instruction was the independent variable. All sessions were taken across eight preferred items. Thus, through observation and data records, the effects of treatment on communicative behaviours were assessed.

\section{PROCEDURE}

\section{Baseline}

Before gestural instruction, frequencies of motoric gesture and deictic gesture behaviours were assessed in this baseline phase. During this phase, Jamie was seated next to the investigator. A preferred item (a toy, food, or drink) was presented on a desk in a separate room, classroom or home setting. When Jamie tried to get a favourite item, the investigator gave him a tiny piece of food (or drink) to taste or a toy to play with for 3 seconds and then moved the item up to a higher counter which Jamie could not reach without assistance. Then, the investigator asked him "Jamie, what do you want?" If Jamie pointed to the preferred item, such as food or a toy with his index finger within 5 seconds, the investigator would give him a larger piece of food (or drink) to taste or a toy to play with for 10 seconds. If an incorrect response or no response occurred within 5 seconds of the start of the interactive episode, the trial was terminated. The investigator provided eight preferred items three times in each probe for observation of Jamie's communicative behaviour. The student was exposed to baseline condition until his data were stable. 


\section{Intervention}

During this phase, a treatment procedure, gestural instruction was applied to Jamie's communicative responses. First, Jamie was seated next to the investigator. A preferred item (a toy, food, or drink) was presented on a desk in a separate classroom. When Jamie tried to get a favourite item, the investigator gave him a tiny piece of food (or drink) to taste or a toy to play with for 3 seconds and then moved the item up to a higher counter which Jamie could not reach without assistance. Second, the investigator asked him "Jamie, what do you want?" If Jamie pointed to the preferred item, such as food or a toy with his index finger within 5 seconds, the investigator would give him a larger piece of food (or drink) to taste or a toy to play with for 10 seconds. In contrast, if Jamie couldn't point to the favorite item after 5 seconds elapsed, the investigator would model a correct deictic gestural response. If Jamie did not imitate the response, the investigator physically prompted him to exhibit the gestural response. Following this correction procedure, the trial was presented again. If the child made the correct gestural response, verbal praise was provided and the trial was terminated. If, again, an incorrect response occurred, the correction procedure was re-introduced. This process continued until the child independently exhibited the correct gestural response within 5 seconds of the start of the interactive episode. The prompts were faded until Jamie's percentage of this deictic gesture behaviour was above $80 \%$ lasting three sessions. Before formal evaluation of Jamie's communicative responses in each session, training and practice of deictic gesture proceeded for twenty minutes. Assessments and observation of Jamie's communicative behaviours were conducted three times a week almost at the same time each day.

\section{Follow-up.}

The follow-up procedures were the same as those conducted in the baseline phase. The gestural instruction was no longer provided during this phase. Two weeks after the intervention phase, the investigator conducted one follow-up session to assess the effects of maintenance of gestural instruction.

\section{Social validity}

At the end of communication training, teachers and parents were interviewed to examine the social validity of these treatment effects. 


\section{RESULTS}

Figure 1 shows the results of Jamie's gestural instruction across three separate settings. In the separate room setting, Jamie's mean percentage of motoric gesture responses occurring during the baseline was $88 \%$ (range, $81 \%$ to $91 \%$ ). After 5 sessions of teaching him appropriate gestural communication to request a preferred item, his aberrant motoric behaviour was decreased to a mean percentage of $54 \%$ (range, $8 \%$ to $83 \%$ ). Therefore, the investigator began to teach him appropriate gestural communication to request a preferred item in his classroom. In total, after 15 sessions of teaching Jamie appropriate gestural communication in this separate room setting, his aberrant motoric behaviour was decreased to a mean percentage of $19 \%$ (range, $0 \%$ to $83 \%$ ), and to a mean percentage of zero $\%$ in the last seven sessions. In contrast, Jamie's mean percentage of motoric gesture responses occurring in his classroom during the baseline probing phase was $84 \%$ (range, $71 \%$ to $91 \%$ ). After 3 sessions of teaching him appropriate gestural communication to request a preferred item, his aberrant motoric behaviour dropped to a mean percentage of $24 \%$ (range, $4 \%$ to $50 \%$ ). Therefore, the investigator began to teach him appropriate gestural communication to request a preferred item in the home setting. In total, after 10 sessions of teaching him appropriate gestural communication in his classroom, his aberrant motoric behaviour was decreased to a mean percentage of $8 \%$ (range, $0 \%$ to $50 \%$, and to a mean percentage of zero $\%$ in the last five sessions. In the home setting, Jamie's mean percentage of motoric gesture responses occurring during the baseline probing phase was $89 \%$ (range, $83 \%$ to $96 \%$ ). After 7 sessions of appropriate gestural instruction in this setting, his aberrant motoric behaviour decreased to a mean percentage of $17 \%$ (range, $0 \%$ to $83 \%$ ), and almost near zero $\%$ in the last four sessions.

On the other hand, Jamie's deictic gesture behaviours showed an inverse pattern to those motoric gesture behaviours across the three different settings. In the separate room setting, Jamie's mean percentage of deictic gesture responses occurring during the baseline was $0 \%$ (range, $0 \%$ to $0 \%$ ). After 15 sessions of teaching him appropriate gestural communication to request a preferred item, his appropriate deictic behaviour was increased to a mean percentage of $73 \%$ (range, $0 \%$ to $100 \%$ ), and to a mean percentage of $100 \%$ in the last seven sessions. 


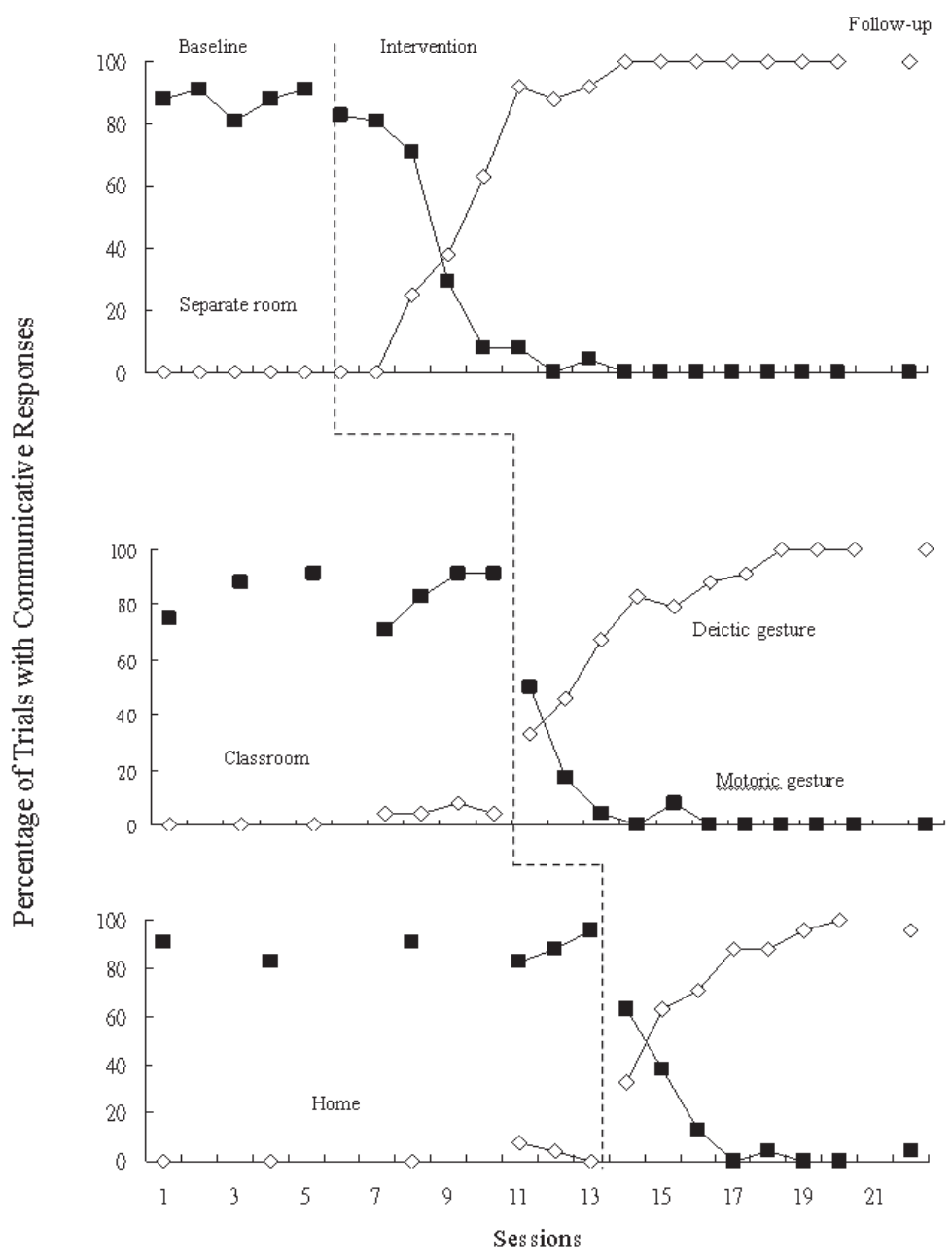

Figure 1. Percentage of communicative responses for Jamie, across three settings

In contrast, Jamie's mean percentage of deictic gesture responses occurring in his classroom during the baseline probing phase was $3 \%$ (range, $0 \%$ to $8 \%$ ). After 10 sessions of teaching him gestural communication in his classroom, his appropriate deictic behaviour was increased to a mean percentage of $79 \%$ (range, $33 \%$ to $100 \%$ ), and to a mean percentage of $100 \%$ in the last three sessions. In the home setting, Jamie's mean percentage of deictic gesture responses occurring during the baseline probing phase was $2 \%$ (range, $0 \%$ to $8 \%$ ). After 7 
sessions of gestural instruction in this setting, his appropriate deictic behaviour was increased to a mean percentage of $77 \%$ (range, $33 \%$ to $100 \%$ ).

As for the follow-up sessions, Jamie's communicative behaviour was reassessed again after two weeks. The behavioural data from Figure 1 indicated that percentages of motoric gesture responses were $0 \%$ in the separate room, $0 \%$ in the classroom, and $4 \%$ in the home setting, respectively. In contrast, Jamie's deictic gesture responses were $100 \%$ in the separate room, $100 \%$ in the classroom, and 96\% in the home setting, respectively.

Finally, both teachers and parents interviewed showed a positive attitude toward the gesture instruction, and confirmed progression on the deictic communication performance of this student. After the experiment, Jamie's teachers affirmed they would continue to use gesture instruction to enhance his communication abilities.

The results from this experimental intervention suggest that gesture instruction could effectively increase the rates of deictic gesture responses and decrease the rates of inappropriate motoric responses. Additionally, treatment effects of gestural instruction could be successfully maintained for at least two weeks. The satisfactory responses from Jamie's parents and teachers further confirm the social validity of gestural instruction on improving deictic communication for Jamie in both home and school settings.

\section{DISCUSSION}

Results of the current study demonstrate that gesture instruction could successfully increase Jamie's levels of deictic gesture behaviour and decrease his levels of inappropriate motoric behaviour. Jamie used little or no gestural communication prior to the intervention. With the introduction of gestural instruction that contained modeling, prompting, and reinforcement, Jamie learned to use deictic gestural responses in the presence of preferred stimuli presented by the investigator during an interactive episode. This is consistent with Buffington et al. (1998), and Carr and Kemp (1989) who also found that gesture instruction could effectively enhance the levels of deictic gestural communication and reduce inappropriate communicative behaviour in children with autism. For example, Carr and Kemp's (1989) study showed that 
children with autism often used contact communication to request adults' assistance during baseline. After an intervention of deictic gestural instruction, contacting behaviour gradually disappeared and was substituted by appropriate deictic behaviour.

Generalization of the gestural behaviour from the training setting to a novel setting was also assessed in the current experiment. The findings suggest that generalization of the trained responses did not occur in the presence of preferred stimuli presented in a novel setting. For example, Jamie's communicative behaviour was dramatically changed after initial introduction of gestural intervention at the separate room setting, but at the same time, remained almost unchanged without intervention during a classroom or home setting. These results are inconsistent with the findings of Buffington et al. (1998) who conducted gestural communication training to four children with autism and found the generalization of verbal and gestural responses from the training setting to a novel classroom setting. It could be that participants in Buffington et al.'s study had better imitation and verbal expression abilities and therefore, found it easier to discriminate between the different stimuli and improve communicative responses between novel settings. Another possible reason why our participant had difficulty with generalization might be that he often stereotyped in everything in the settings.

Finally, the social validity measures taken in this study suggest that the participant's behaviour appeared more socially appropriate after treatment. Teachers and parents consistently rated posttreatment video scenes as more socially appropriate than baseline video scenes. However, the follow-up sessions were conducted two weeks after intervention in the current study (see Figure 1). At best, it could demonstrate the short term effectiveness of withdrawing treatments. The long term effects of maintenance in gestural instruction still remain uncertain. Extended analyses and studies to support such maintenance effects are needed.

\section{REFERENCES}

Attwood, A., Frith, U., \& Hermelin, B. (1988). The understanding and use of interpersonal gestures by autistic and down's syndrome children. Journal of Autism and Developmental Disorders, 18 (2), 241-257. 
Baron-Cohen, S. (1989). Perceptual role-taking and protodeclarative pointing in autism. British Journal of Developmental Psychology, 7, 113-127.

Buffington, D. M., Krantz, P. J., McClannahan, L. E., \& Poulson, C. L. (1998). Procedures for teaching appropriate gestural communication skills to children with autism. Journal of Autism and Developmental Disorders, 28 (6), 535-544.

Carr, E. G., \& Kemp, D. C. (1989). Functional equivalence of autistic leading and communicative pointing: Analysis and treatment. Journal of Autism and Developmental Disorders, 19 (4) , 561-578.

Chuang, M-F. (2001). The effects of alternative communication training on communication ability and problem behaviour of autistic children with low functionality.

Curcio, F. (1978). Sensorimotor functioning and communication in mute autistic children. Journal of Autism and Childhood Schizophrenia, 8 (3), 281-292.

DeLeon, I. G., \& Iwata, B. A. (1996). Evaluation of a multiplestimulus presentation format for assessing reinforcer preferences. Journal of Applied Behaviour Analysis, 29, 519-533.

Garfin, D. G., \& Lord, C. (1986). Communication as a social problem in autism. In E. Schopler \& G. B. Mesibov (Eds.), Social behaviour in autism (pp. 133-149). New York: Plenum Press.

Hetzroni, O. E., \& Shalem, U. (2005). From logos to orthographic symbols: A multilevel fading computer program for teaching nonverbal children with autism. Focus on Autism and other Developmental Disabilities, 20, 201-212.

Kasari, C., Sigman, M., Mundy, R, \& Yirmiya, N. (1990). Affective sharing in the context of joint attention interactions of normal, autistic, and mentally retarded children. Journal of Autism and Developmental Disorders, 20, 87-100.

Kazdin, A. E. (1982). Single-case research designs. New York: Oxford University Press.

Keen, D., Sigafoos, J., \& Woodyatt, G. (2005). Teacher responses to the communicative attempts of children with autism. Journal of Developmental and Physical Disabilities, 17, 19-33.

Koegel, R. L, \& Frea, W D. (1993). Treatment of social behaviour in autism through the modification of pivotal social skills. Journal of Applied Behaviour Analysis, 26, 369-377. 
Landry, S. H., \& Loveland, K. A. (1989). The effect of social context on the functional communication skills of autistic children. Journal of Autism and Developmental Disorders, 19, 283-299.

Loveland, K. A., \& Landry, S. H. (1986). Joint attention and language in autism and developmental language delay. Journal of Autism and Developmental Disorders, 16(3), 335-349.

Mundy, P., Sigman, M., \& Kasari, C. (1990). A longitudinal study of joint attention and language development in autistic children. Journal of Autism and Developmental Disorders, 20, 115-128 .

Mundy, P., Sigman, M., Ungerer, J., \& Sherman, T (1986). Defining the social deficits of autism: The contribution of nonverbal communication measures. Journal of Child Psychology and Psychiatry, 27, 657-669.

Paparella, T., \& Kasari, C. (2004). Joint attention skills and language development in special needs populations: Translating research to practice. Infants and Young Children, 17, 269-280.

Rosenquest, B. B. (2003). 0-2 years: First signs. Scholastic Parent and Child, 10 (4), 59-60.

Schopler, E., \& Bristol, M. M. (1993). Preschool issues in autism. New York: Plenum Press.

Sigman, M., Mundy, P., Sherman, T., \& Ungerer, J. (1986). Social interactions of autistic, mentally retarded and normal children and their caregivers. Journal of Child Psychology and Psychiatry and Allied Disciplines, 27, 647-656.

Smith, I. M., \& Bryson, S. E. (1998). Gesture imitation in autism I : Nonsymbolic postures and sequences. Cognitive Neuropsychology, 15(68), 747-770.

Stone, W. L., Ousley, O.Y., Yoder, P. J., Hogan, K. L., \& Hepburn, S. L. (1997). Nonverbal communication in two- and three-year-old children with autism. Journal of Autism and Developmental Disorders, 27 (6 ), 677-696.

Wetherby, A. M., \& Prutting, C. A. (1984). Profiles of communicative and cognitive- social abilities in autistic children. Journal of Speech and Hearing Research, 27, 364-377. 